\title{
Effect of Agmatine Sulfate on Modulation of Matrix Metalloproteinases via PI3K/Akt-1 in HT1080 Cells
}

\author{
HYEJEONG KIM and MOON-MOO KIM \\ Department of Applied Chemistry, Dong-Eui University, Busan, Republic of Korea
}

\begin{abstract}
Background/Aim: The purpose of this study was to investigate the mechanism by which agmatine sulfate induces an anti-metastatic effect in human HT1080 fibrosarcoma cells, by affecting matrix metalloproteinases (MMPs). Materials and Methods: For the experiments, we used a non-toxic concentration of agmatine, below $512 \mu \mathrm{M}$, that was determined using an MTT assay. The effect of agmatine sulfate on metastasis was gelatin zymography, western blot, immunofluorescence staining and cell invasion assay. Results: Agmatine sulfate inhibited MMP-2 activity stimulated by phenazine methosulfate (PMS). Furthermore, the expression level of MMP-2 stimulated by PMS, was decreased, but the expression level of TIMP-1 was increased in the presence of agmatine sulfate. Moreover, it was observed that the expression levels of ERK and p38 were increased, but those of PI3K and Akt-1 associated with the modulation of MMP-2 were decreased in this study. Furthermore, agmatine sulfate decreased the invasion level of human fibrosarcoma cells stimulated by VEGF. Conclusion: These results suggest that agmatine sulfate could inhibit metastasis through inhibition of MMP-2 via the PI3K/Akt-1 signaling pathway.
\end{abstract}

Cancer, among several other diseases, is a major cause of death with a rapidly increasing mortality rate. The majority of cancer-related deaths are due to cancer tumor metastasis (1). Recently, the inhibition of tumor invasion and metastasis has been extensively studied in order to treat and prevent cancer. For cancer invasion and metastasis, cancer cells require a variety of interactions with the extracellular matrix surrounding the tumor. The main group of proteolytic

Correspondence to: Moon-Moo Kim, Department of Applied Chemistry, Dong-Eui University, Busan, 614-714, Republic of Korea. Tel: +82 51890-1511, Fax: +82 518902620, e-mail: mmkim@deu.ac.kr

Key Words: Agmatine suflate, MMPs, HT1080, metastasis, PI3K, Akt-1. enzymes related to tumor invasion and metastasis are matrix metalloproteinases (MMPs). They cleave polypeptide bonds in extracellular matrix proteins such as collagen, elastin, fibronectin, proteoglycan and laminin (2, 3). MMPs implicated in the invasive and metastatic spread of cancer include gelatin A (MMP-2) and gelatin B (MMP-9). The activity of MMPs is modulated by the tissue inhibitors of metalloproteinases (TIMPs). In particular, TIMP-1 regulates the inhibition of MMP-2 activity $(4,5)$. The expression of MMP-2, related to metastasis, was reported to be regulated via the MAPK signaling pathway including extracellular signal-regulated kinases (ERK), c-jun N-terminal kinases (JNK) and p38. The MAPK signaling pathway has been reported to regulate cell growth, apoptosis and tumor metastasis $(6,7)$. In addition, the activation of the phosphatidylinositol-3-kinase (PI3K)/Akt signaling pathway has been known to regulate MMPs and promote tumor invasion. The PI3K/Akt signaling pathway plays a major role in cellular processes such as metastasis of tumor cells (8).

However, the natural compounds capable of completely inhibiting MMPs associated with metastasis have not been reported until now. Therefore, we examined whether Brunfelsia uniflora (manaca) extract known to have antiaging effects may inhibit metastasis and cancer invasion. Brunfelsia uniflora extract has been known to contain agmatine, alkalods-franciscain, manainm and scopoletin (9, 10). Previous studies have shown that agmatine sulfate exerts an anti-proliferative effect in cancer and may be effective in the treatment of sepsis (11). Therefore, in this study we investigated the inhibitory effect of agmatine sulfate on the modulation of MMP-2 via the PI3K/Akt signaling pathway.

\section{Materials and Methods}

Materials. Dulbecco's modified Eagle's medium (DMEM), trypsinEDTA, penicillin/streptomycin/ amphotericin (10000 U/ml, 10000 $\mathrm{g} / \mathrm{ml}$, and $2500 \mathrm{~g} / \mathrm{ml}$, respectively) and fetal bovine serum (FBS) were obtained from Gibco BRL, Life Technologies (NY, USA). HT1080 cells were purchased by ATCC. MTT reagent, agmatine sulfate and other materials were purchased from Sigma Chemical Co. (St. Louis, MO, USA). 
Cell line and culture. Cell lines were separately grown as monolayers at $5 \% \mathrm{CO}_{2}$ and $37^{\circ} \mathrm{C}$ humidified atmosphere using appropriate media supplemented with $5 \%$ fetal bovine serum, $2 \mathrm{mM}$ glutamine and $100 \mathrm{~g} / \mathrm{ml}$ penicillin-streptomycin. DMEM was used as the culture medium for the HT1080 cell line. Cells were passaged 3 times a week by treating with trypsin-EDTA.

MTT assay. Cytotoxic levels of agmatine sulfate on HT1080 cells were measured using 3-(4,5-dimethyl-2-yl)-2,5-diphenyltetrazolium bromide (MTT) method as described by Hansen et al. (12).

Gelatin zymography. Activation of MMPs were determined by zymography as described previously (13) in the presence or absence of agmatine sulfate. Conditioned medium containing same amount of total proteins was loaded into each well of a polyacrylamide gel containing $1.5 \mathrm{mg} / \mathrm{ml}$ gelatin and electrophoresed under non-reducing conditions. Depending on the purpose, different protein amounts for each experiment were used for gelatin zymography analysis. Gelatinolytic bands were observed as clear zones against the blue background and the intensity of the bands was estimated using ImageMaster Software (Amersham Pharmacia Bioscience, NJ, US).

Western blot analysis. Western blot analysis was performed according to standard procedures. Cell lysates or nuclear proteins were resolved on a 4-20\% Novex ${ }^{\circledR}$ gradient gel (Invitrogen, US), electro transferred onto a nitrocellulose membrane and blocked with $10 \%$ skim milk. The primary antibodies including anti-MMP-2, anti-TIMP-1, anti- $p$-ERK, anti-p-p38, anti- $p$-JNK, anti- $p$-PI3K, antiAkt- 1 and anti- $\beta$-actin and their secondary antibodies (Santa Cruz BiotechnologufInc., CA, US) were used to detect respective proteins using a chemiluminescent ECL assay kit (Amersham Pharmacia Biosciences, NJ, US) according to the manufacturer's instructions. Protein bands were visualized using AlphaEase ${ }^{\circledR}$ gel image analysis software (Alpha Innotech, CA, US).

Analyses of proteins expression using Immunofluorescence assay. HT1080 cells were seeded in slide chambers and incubated overnight at $37^{\circ} \mathrm{C}$. Then, cells were treated with agmatine sulfate and stimulated with PMS as a negative control. After $24 \mathrm{~h}$ of incubation, cells were fixed with $10 \%$ formalin for $15 \mathrm{~min}$ at room temperature followed by permeabilization with PBS containing $0.5 \%$ tween $20(0.5 \%$ PBS T-20) and washing for three times with $0.1 \%$ PBS T-20. The cells were preconditioned with 5\% Donkey normal serum and analysed by immunofluorescence staining using a primary antibody (MMP-2) (1:500) for $24 \mathrm{~h}$ at room temperature. Afterwards, the cells were washed with $0.1 \%$ PBS T-20 three times for $5 \mathrm{~min}$, respectively and treated with secondary antibodies (donkey anti- rabbit conjugated FITC) (FITC 1:200) at room temperature for $1 \mathrm{~h}$. The cells were then washed with $0.1 \%$ PBS T20 three times and PBS once for $5 \mathrm{~min}$, respectively. Finally, the slide was stained with DAPI solution and examined using Axio Scope A1 Microscope (Carl Zeiss, Goettingen, Germany).

In vitro cell invasion assay. Cell invasion assay was performed according to the protocol from Chemicon ${ }^{\circledR}$. The Cell Invasion Assay Kit (ECM550) utilizes an Invasion Chamber, which consists of a 24-well tissue culture plate and 12 cell culture inserts. For quantification stained cells were dissolved in $10 \%$ acetic acid and a consistent amount of the dye/solute mixture was transferred to a 96well plate for colorimetric reading of OD at $560 \mathrm{~nm}$.
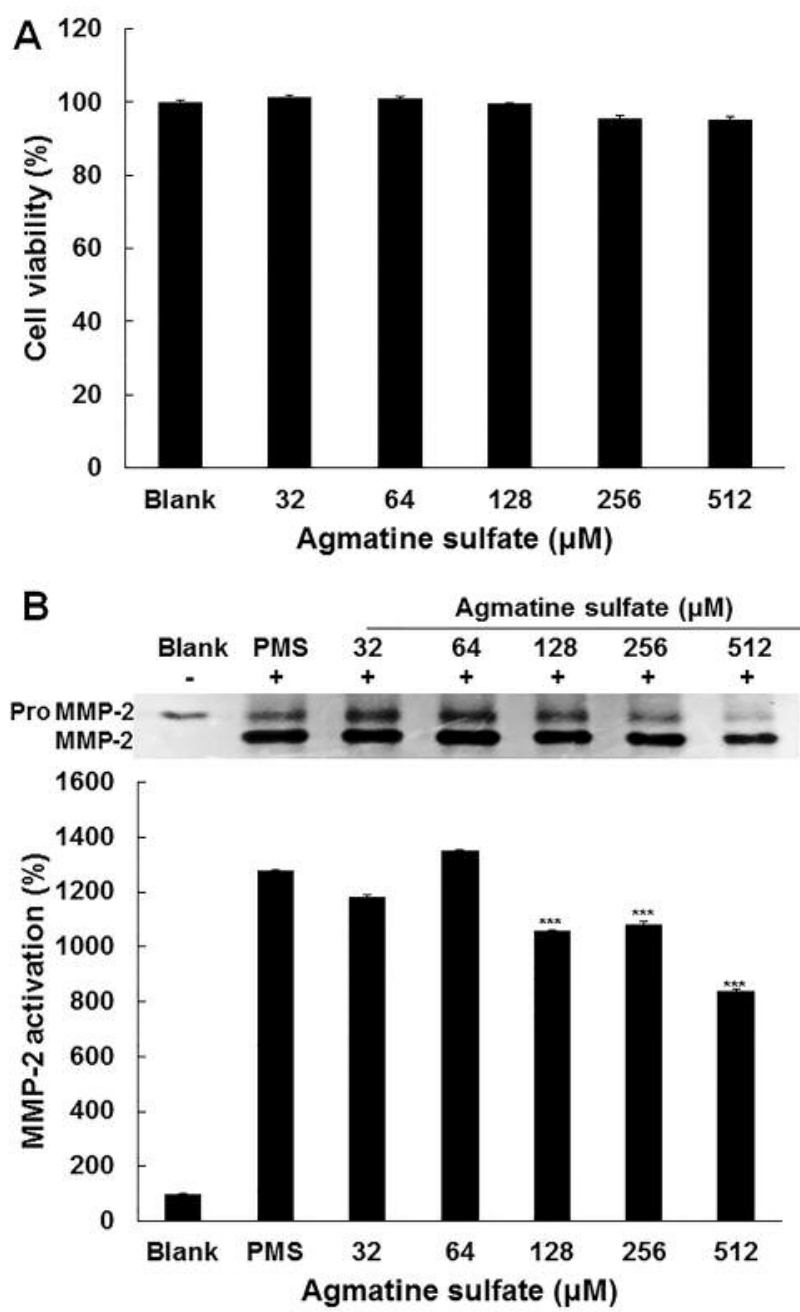

Figure 1. Effect of agmatine sulfate on viability and activity of MMP-2 in HT1080 cells. (A) HT1080 cells were treated with agmatine sulfate at 32, 64, 128, 256 and $512 \mu \mathrm{M}$. Cell viability was determined by MTT assay after $24 \mathrm{~h}$. (B) The cells stimulated with $2 \mu \mathrm{M}$ of PMS to induce MMPs expression were treated with agmatine sulfate at 32, 64, 128, 256 and $512 \mu M$ under serum-free conditions for 72 h. MMP-2 activity in conditioned media were determined by gelatin zymography. Data are given as means of values $\pm S . D$. from three independent experiments. Level of significance was identified statistically $(* * * p<0.001)$ using the Student's t-test.

Statistics. Data were analyzed using the Student's $t$-test for paired data (comparison with control group) and MEGFL. Data are given as means of values \pm S.D. from three independent experiments $\left({ }^{*} p<0.05, * * p<0.01, * * * p<0.001\right)$.

\section{Results}

Effect of agmatine sulfate on cytotoxicity and MMP-2 activity in HT1080 cells stimulated with PMS. In order to determine the non-cytotoxic concentration of agmatine 


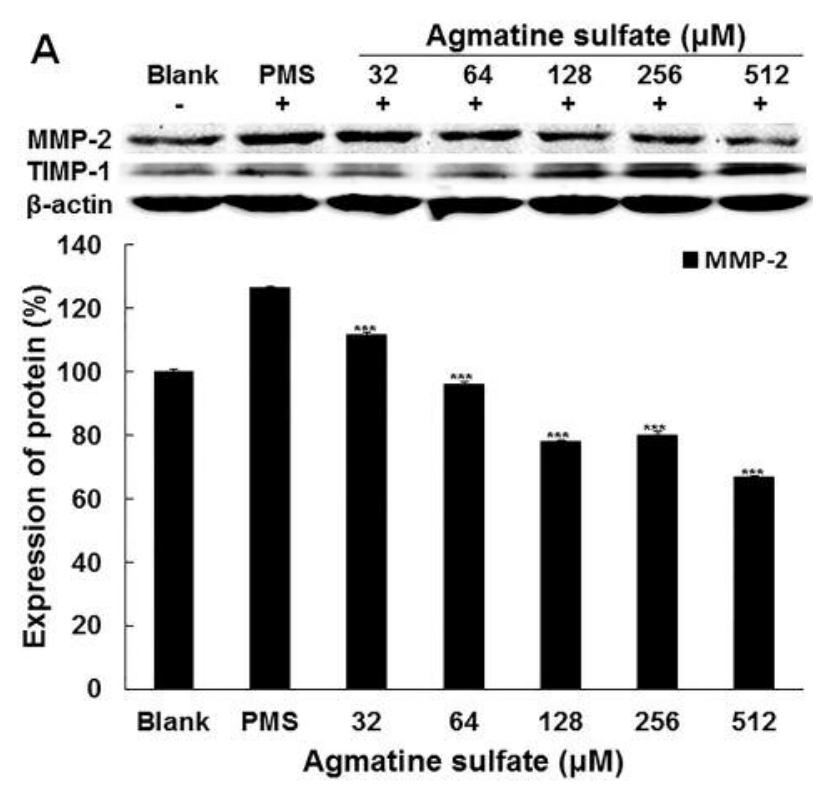

B

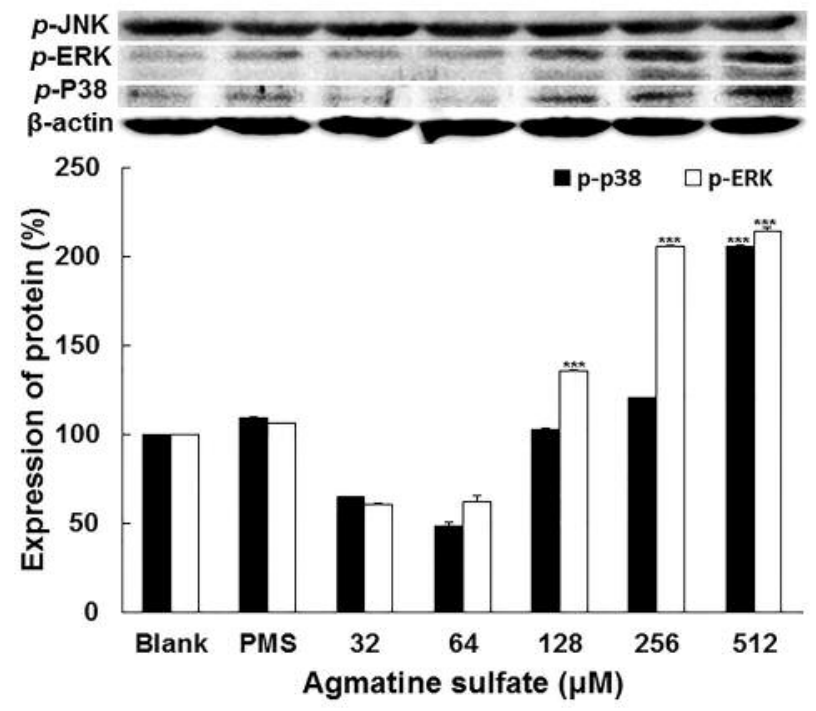

Figure 2. Effect of agmatine sulfate on MMP-2 and MAPK expression related to metastasis in $H T 1080$ cells. The cells were treated with agmatine sulfate at 32, 64, 128, 256 and $512 \mu \mathrm{M}$ prior to stimulation of cells with $2 \mu M$ PMS for $24 \mathrm{~h}$. Western blot analysis of cell lysates was performed using antibodies as indicated. The expression level of $\beta$-actin was used as a control for normalization of target proteins. Data are given as means of values $\pm S . D$. from three independent experiments. Level of significance was identified statistically $(* * * p<0.001)$ using Student's t-test.

sulfate on HT1080 cells, an MTT assay was carried out. The human fibrosarcoma cells were treated with agmatine sulfate at 32, 64, 128, 256 and $512 \mu \mathrm{M}$ for $24 \mathrm{~h}$. As shown in Figure 1A, agmatine sulfate at all concentrations did not exert a

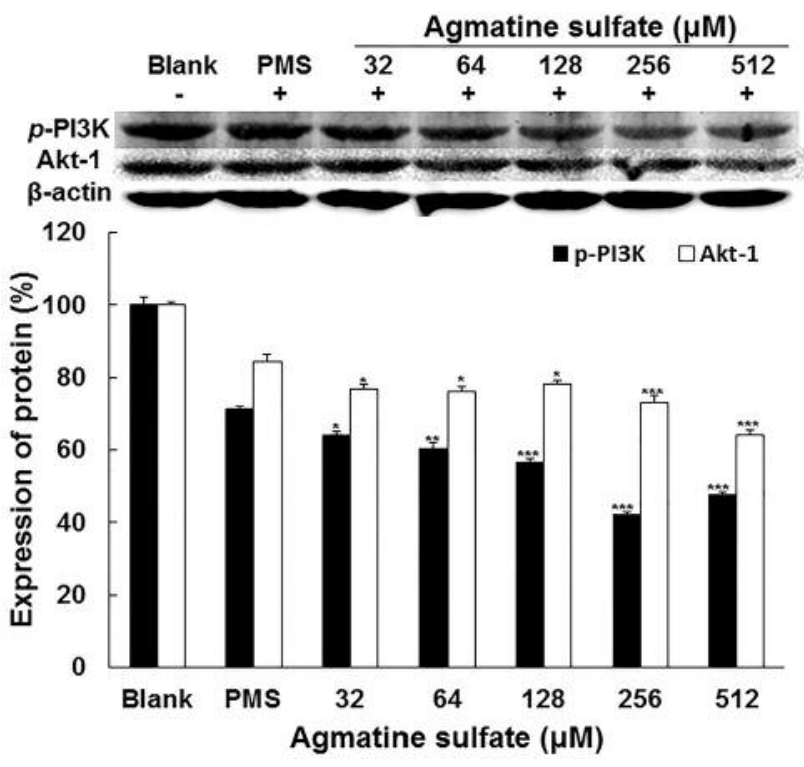

Figure 3. Effect of agmatine sulfate on PI3K/Akt-1 expression related to metastasis in HT1080cells. The cells were treated with agmatine sulfate at 32, 64, 128, 256 and $512 \mu \mathrm{M}$ prior to stimulation of cells with $2 \mu M$ PMS for $24 \mathrm{~h}$. Western blot analysis of cell lysates was performed using antibodies as indicated. The expression level of $\beta$-actin was used as a control for normalization of target proteins. Data are given as means of values $\pm S . D$. from three independent experiments. Level of significance was identified statistically $\left({ }^{*} p<0.05 ; \quad * * p<0.01\right.$; $* * * p<0.001)$ using Student's t-test.

cytotoxic effect on fibrosarcoma cells. Therefore, a concentration of agmatine sulfate of less than $512 \mu \mathrm{M}$ was used for further study in human fibrosarcoma cells. In the next experiment, gelatin zymography was performed in HT1080 cells to examine the effect of agmatine on MMP-2 activity. The cells were cultured for $72 \mathrm{~h}$ after stimulation of cells with phenazine methosulfate (PMS) that generates intracellular $\mathrm{H}_{2} \mathrm{O}_{2}$ following agmatine sulfate treatment at the indicated concentration. As shown in Figure 1B, it was observed that the inactive pro MMP-2 form, was converted into MMP-2, an active form, by treatment with PMS. Agmatine sulfate at $128 \mu \mathrm{M}$ inhibited the activity of MMP2 by $200 \%$ compared with the PMS treated human fibrosarcoma cells. This result suggests that agmatine sulfate could inhibit metastasis.

Effect of agmatine sulfate on the expression of MMP-2, TIMP-1 and MAPK related to metastasis in HT1080 cells. The effect of agmatine sulfate on the expression levels of MMP-2, TIMP-1 and MAPK in HT1080 cells was investigated using western blot analysis in order to explore the signaling pathway of MMP-2. As shown in Figure 2A, agmatine sulfate inhibited the expression level of MMP-2 in a dose-dependent manner compared to the PMS-treated cells. 


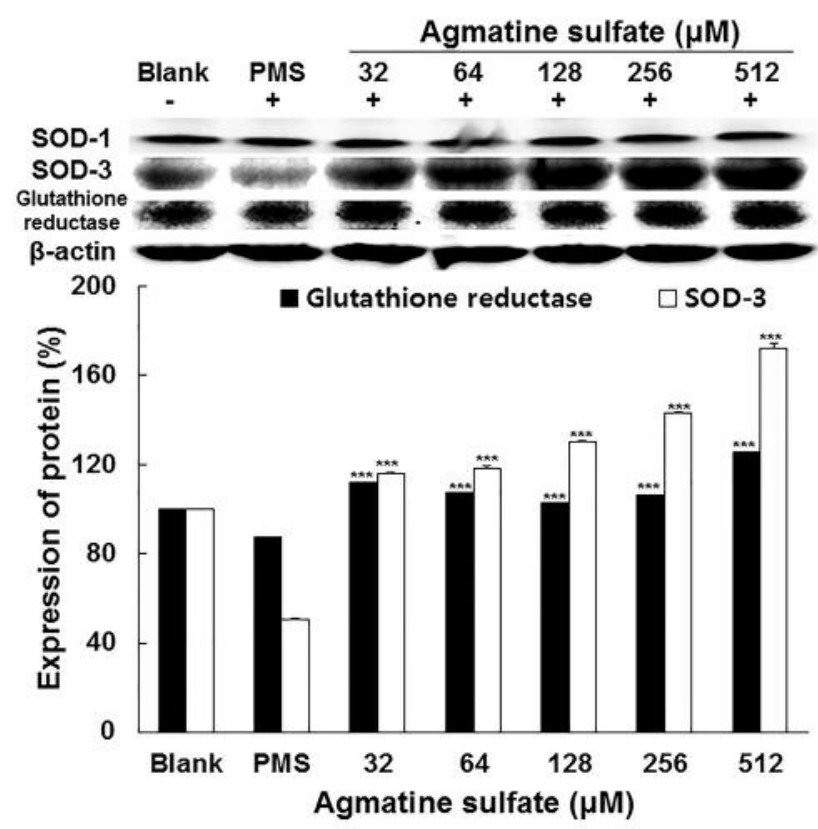

Figure 4. Effect of agmatine sulfate on protein expression related to antioxidant enzyme in HT1080 cells. The cells were treated with agmatine sulfate at 32, 64, 128, 256 and $512 \mu \mathrm{M}$ prior to stimulation of cells with $2 \mu M$ PMS for $24 \mathrm{~h}$. Western blot analysis of cell lysates was performed using antibodies as indicated. The expression level of $\beta$-actin was used as a control for normalization of target proteins. Data are given as means of values $\pm S$.D. from three independent experiments. Level of significance was identified statistically $\left({ }^{* * *} p<0.001\right)$ using the Student's t-test.

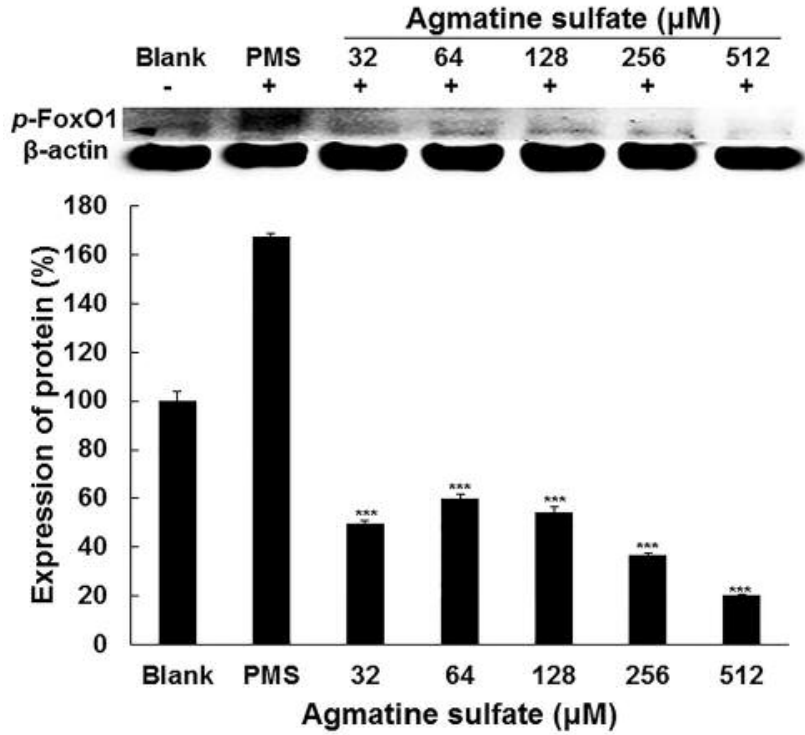

Figure 5. Effect of agmatine sulfate on the level of p-FoxO1 expression in nucleus. HT1080 cells were pre-treated with different concentrations of agmatine sulfate for $1 \mathrm{~h}$ and followed for $24 \mathrm{~h}$ of incubation. After nucleus extraction, equal amounts of protein in the nuclear lysates were electrophoresed and level of p-FoxO1 expression was determined. The expression level of $\beta$-actin was used as a control for normalization of target proteins. Data are given as means of values $\pm S . D$. from three independent experiments. Level of significance was identified statistically $(* * *, p<0.001)$ using Student's t-test.
Furthermore, agmatine sulfate increased the expression of TIMP-1 in human fibrosarcoma cells treated with a concentration above $128 \mu \mathrm{M}$. As shown in Figure 2B, agmatine sulfate did not exert any effect on the expression of $p$-JNK in human fibrosarcoma cells. However, it was observed that agmatine sulfate increased the expression levels of $p$-ERK and $p$-p38. Therefore, these results indicate that inhibition of MMP expression by agmatine sulfate was modulated by the increase of TIMP-1 expression.

Effect of agmatine sulfate on the expression of p-PI3K and AKT-1 in HT1080 cells. On the other hand, in order to examine the effect of agmatine sulfate on the $p$-PI3K and Akt-1 signaling pathway related to MMP-2, the expressions of PI3K and Akt-1 were analyzed by western blotting in HT1080 cells. As shown in Figure 3, agmatine sulfate decreased the expression levels of $p$-PI3K and Akt-1 in a dose-dependent manner using concentrations above $32 \mu \mathrm{M}$. Therefore, these results indicate that the inhibition of MMP expression by agmatine sulfate could be regulated by the activation of PI3K/Akt-1.
Effect of agmatine sulfate on the expression of SOD-1, SOD3 and glutathione reductase related to antioxidant activity in HT1080 cells. Because PMS produces $\mathrm{H}_{2} \mathrm{O}_{2}$ in cells, the effect of agmatine sulfate on the expression of antioxidant proteins such as SOD-1, SOD-3 and glutathione reductase was investigated using western blot analysis in HT1080 cells. As shown in Figure 4, agmatine sulfate had no effect on the expression of SOD-1. However, agmatine sulfate increased the expression of SOD-3 in human fibrosarcoma cells by $65 \%$ compared to the PMS-treatment group. Especially, the expression of glutathione reductase was increased in a dose-dependent manner by agmatine sulfate. Therefore, these results indicate that agmatine sulfate could affect the expression of antioxidant enzymes.

Effect of agmatine sulfate on the expression of p-FoxO1 in nucleus. Forkhead-box O (FoxO1) modulates the expression of antioxidant enzymes such as SOD and glutathione reductase in the nucleus. However, p-FoxO1 inhibits the expression of antioxidant enzymes. The effect of agmatine sulfate on the expression of p-FoxO1 in the nucleus was 


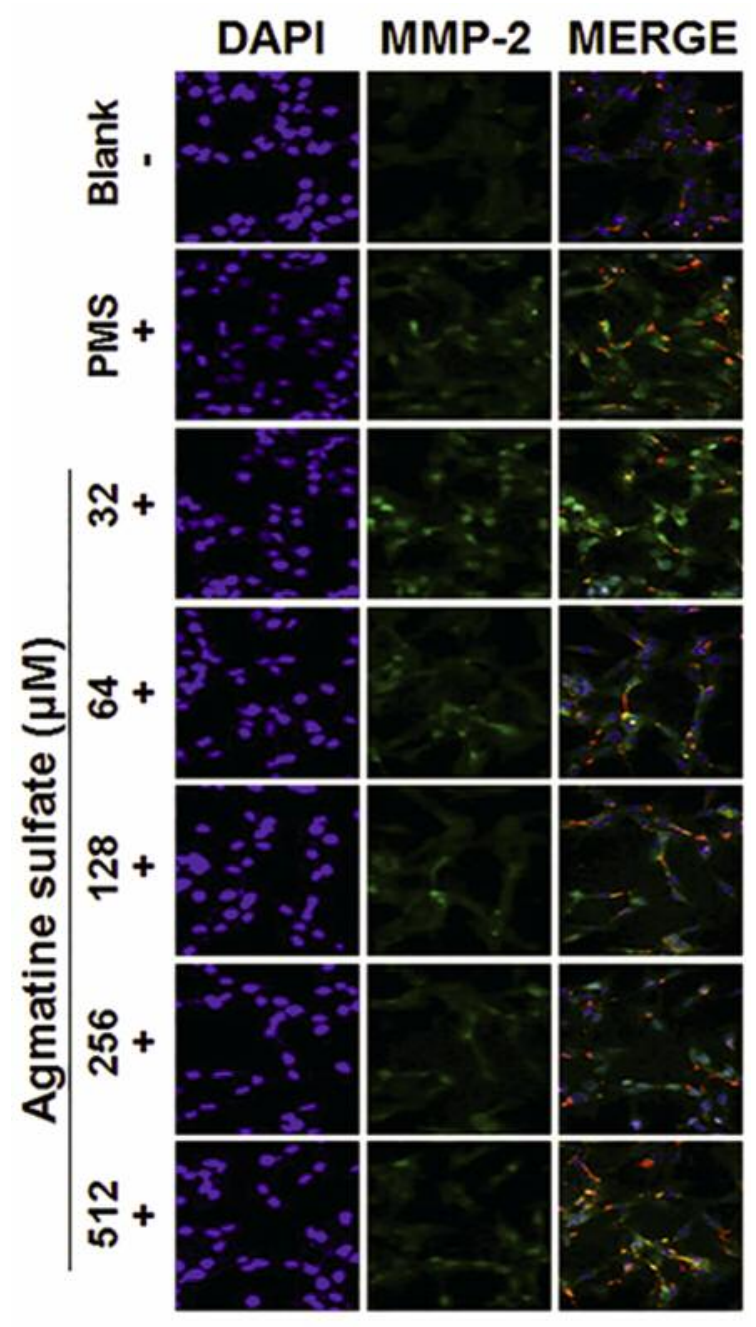

Figure 6. Effect of agmatine sulfate on MMP-2 expression in HT1080 cells using immunofluorescence staining analysis. HT1080 cells were cultured in the presence of PMS or agmatine sulfate and MMP-2 expression was detected with a mouse polyclonal MMP-2 antibody. MMP-2 localized to the cell cytosol. MMP-2 was observed in green color and nucleus was observed in blue. The merged image was observed in yellow green color using fluorescence microscopy at $200 \mathrm{X}$ of magnification.

investigated by western blot analysis. As shown in Figure 5, agmatine sulfate decreased the expression of p-FoxO1 using a concentration above $32 \mu \mathrm{M}$ compared to the PMStreatment group in HT1080 cells. This result indicates that agmatine sulfate could enhance the expression of antioxidant enzymes by regulating FoxO1.

Immunofluorescence analysis for the expression of MMP-2 in HT1080 cells treated with agmatine sulfate. In order to confirm whether agmatine sulfate affects the expression of MMP-2, immunofluorescence staining was carried out in

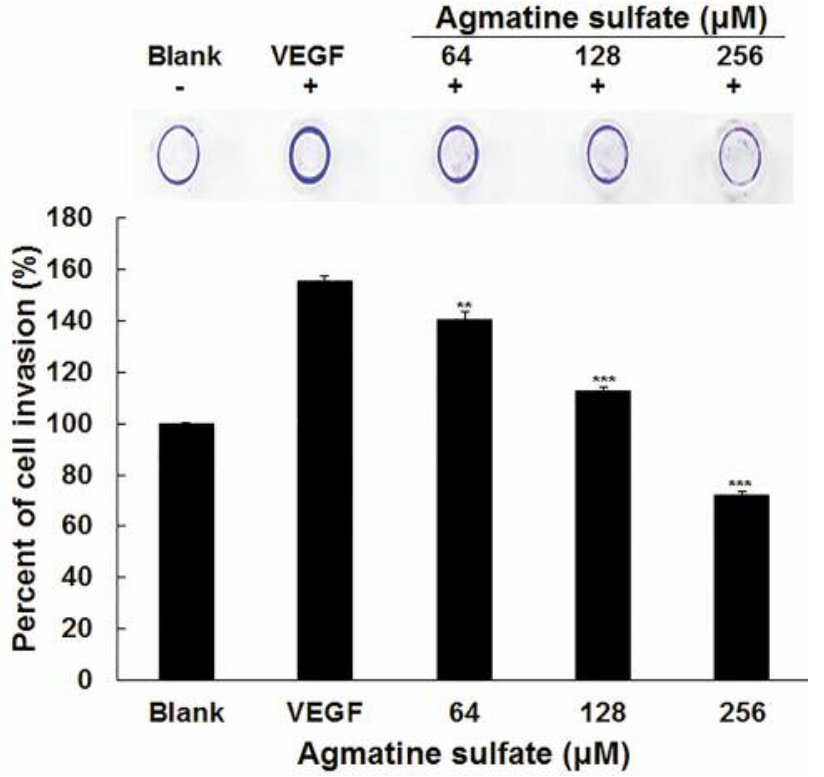

Figure 7. Effects of agmatine sulfate on cell invasion of HT1080 cells. Invasion assay was performed in an invasion chamber, a 24-tissue culture plate with 5-cell culture insert, 8-um pore size of polycarbonate membrane coated with matrigel. HT1080 cells were seeded to polycarbonate membrane. After treatment with agmatine sulfate for $24 \mathrm{~h}$, non-invaded cells were removed using cotton-tipped swab and then the invaded cells were stained with staining solution. Data are given as means of values $\pm S . D$. from three independent experiments. Level of significance was identified statistically $\left({ }^{* *} p<0.01\right.$; $* * * p<0.001)$ using the Student's t-test.

HT1080 cells treated with agmatine sulfate. As shown in Figure 6, MMP-2 protein expression visualized by FITCfluorescence (green) was higher in the PMS treatment group than the blank group. However, agmatine sulfate attenuated MMP-2 protein expression compared with the PMS treatment group in HT1080 cells underimmunofluorescence observation.

Effect of agmatine sulfate on cell invasion in HT1080 cells stimulated with VEGF. When cancer cells lack nutrients, they move into the surrounding tissues and organ by degrading the base membrane and extracellular matrix. Therefore, it was examined whether agmatine sulfate can inhibit cell invasion. As shown in Figure 7, VEGF was used to stimulate cell invasion and cell movement from the upper part of the polycarbonate membrane into the lower part of the membrane. When the cells were pre-treated with agmatine sulfate exposed to VEGF, agmatine sulfate decreased cell invasion in a dose dependent manner. It was also observed that the cells treated with agmatine sulfate at $64 \mu \mathrm{M}$ inhibited cell invasion by $16 \%$ compared to the VEGF group. Therefore, this result provides evidence that agmatine sulfate could inhibit metastasis. 


\section{Discussion}

Inhibition of tumor invasion and metastasis is very important for the treatment of cancer. Recently, it has been reported that natural compounds such as platycodin D (14), paris saponin VII (15) and phloretin (16) inhibit metastasis associated with MMP. Therefore, we screened for natural compounds that inhibit MMPs and cell invasion. In this study, we investigated the effect of agmatine sulfate on metastasis related to the expression MMP-2 in human fibrosarcoma cells (17). In this study, we examined the inhibitory effect of agmatine sulfate on the activity of PMSinduced MMPs using gelatin zymography in HT1080 cells. Agmatine sulfate significantly revealed the inhibitory effect on MMP-2 activation stimulated by PMS which is known as an inducer of intracellular hydrogen peroxide. In a previous study it was reported that human fibrosarcoma cells treated with PMS promoted the activation of proMMP-2 (18). In addition, PMS-induced cell motility is inhibited by MMP-2, which is associated with the inhibition of intracellular levels of ROS. Our results demonstrated that the cells induced by PMS increased activation of MMP-2. Moreover, the effects of agmatine sulfate on the expression levels of the MMP-2 and TIMP-1, major proteins involved in tumor metastasis, were investigated in this study. The expression of MMP-2 in the human fibrosarcoma cells were decreased by agmatine sulfate in a concentration dependent manner. Agmatine sulfate salt also increased the expression level of TIMP-1. Among TIMPs, TIMP-1 is known to inhibit the activity of MMP (19). Our results showed that the inhibitory effect of agmatine sulfate on the activities of MMP-2 is caused by TIMP-1. Moreover, in another study it was reported that MAPK and PI3K/Akt-1 signaling pathway among several other signaling pathways are related to the regulation of MMPs (20). Thus, we aimed to investigate the effects of agmatine sulfate on the MAPK and PI3K/Akt-1 signaling pathways. It was predicted that the MAPKs such as JNK, ERK and p38 are inactivated. However, in this study the effect of agmatine sulfate on the expression of MMP-2 was not found to be associated with $p$-JNK. In contrast, MAPK such as ERK and p38 were activated. Although the inhibition of MMP-2 via the MAPK signaling pathway by agmatine sulfate cannot be clearly explained by this finding, the previous study reported that the expression of $p$-38 and $p$-JNK was increased, and promoted apoptosis. In addition, the expression of $p$-Akt was decreased, which suggested that the anticancer effect is regulated by the PI3K/Akt signaling pathway (21). These results suggest that the modulation of MMP-2 by agmatine sulfate is not affected by the MAPK signaling pathway. On the other hand, the expression levels of PI3K and Akt-1 were downregulated in fibrosarcoma cells. A previous study reported that the activation of MMPs involved in metastasis is modulated by the PI3K/Akt-1 signaling pathway (22). Our results indicated that the MMP-2 expression reduced by agmatine sulfate is not due to the decreased level of PI3K/Akt-1 but due to the MAPK signaling pathway. Thus, our findings suggest that the PI3K/Akt-1 signaling pathway plays an important role in the inhibition of metastasis by agmatine sulfate. In addition, the agmatine sulfate remarkably increased the expression of antioxidant proteins such as glutathione reductase and SOD-3 in HT1080 cells. This evidence suggests that the increase in the expression levels of antioxidant proteins by agmatine sulfate could reduce the level of intracellular hydrogen produced by PMS, which could inhibit the expression of MMP-2. Moreover, we investigated the effect of agmatine sulfate on the expression of $p$-FoxO1 in the nucleus. Agmatine sulfate reduced the expression level of $p$-FoxO1 involved in inhibition of antioxidant gene transcription. This result indicates the relative increase of FoxO1 due to the decrease of $p$-FoxO1 level in the nucleus promoted the nuclear expression of antioxidant enzymes such as SOD and glutathione reductase consistent with a previous report (23). The inhibitory effect of agmatine sulfate is consequently confirmed by immunofluorescence for the expression of MMP-2. In addition, in order to investigate whether agmatine sulfate could inhibit metastasis, an in vitro cell invasion assay was performed with human fibrosarcoma cells. The inhibitory effect of agmatine sulfate on the cell invasion was observed in fibrosarcoma cells. A previous study reported that the inhibition of cell invasion is modulated by the expression of MMP related to metastasis (24). In conclusion, the above results suggest that the activation PI3K/Akt-1 and the increase of TIMP-1 expression in the presence of agmatine sulfate could inhibit the expression of MMP-2 in human fibrosarcoma cells.

\section{Conflicts of Interest}

None of the Authors have any conflicts of interest in regard to this study.

\section{References}

1 Torre LA, Bray F, Siegel RL, Ferlay J, Lortet-Tieulent J, and Jemal A: Global cancer statistics, 2012. CA Cancer J Clin 65: 87-108, 2015.

2 Brown GT and Murray GI: Current mechanistic insights into the roles of matrix metalloproteinases in tumour invasion and metastasis. J Pathol 237: 273-281, 2015.

3 Xue $\mathrm{M}$ and Jackson CJ: Extracellular matrix reorganization during wound healing and its impact on abnormal scarring. Adv Wound Care 4: 119-136, 2015.

4 Wang N, Zhu M, Tsao S-W, Man K, Zhang Z and Feng Y: Upregulation of TIMP-1 by genipin inhibits MMP-2 activities and suppresses the metastatic potential of human hepatocellular carcinoma. PLoS One 7: e46318, 2012. 
5 Jacob A and Prekeris R: The regulation of MMP targeting to invadopodia during cancer metastasis. Front Cell Dev Biol 3: 4, 2015.

6 Wang X-F, Zhou Q-M, Lu Y-Y, Zhang H, Huang S and Su S-B: Glycyrrhetinic acid potently suppresses breast cancer invasion and metastasis by impairing the p38 MAPK-AP1 signaling axis. Exp Opin Ther Targets 19: 577-587, 2015.

7 Aroui S, Aouey B, Chtourou Y, Meunier A-C, Fetoui H and Kenani A: Naringin suppresses cell metastasis and the expression of matrix metalloproteinases (MMP-2 and MMP-9) via the inhibition of ERK-P38-JNK signaling pathway in human glioblastoma. Chem Biol Interact 244: 195-203, 2016.

8 Lee Y-C, Lin H-H, Hsu C-H, Wang C-J, Chiang T-A and Chen $\mathrm{J}-\mathrm{H}$ : Inhibitory effects of andrographolide on migration and invasion in human non-small cell lung cancer A549 cells via down-regulation of PI3K/Akt signaling pathway. European journal of pharmacology 632: 23-32, 2010.

9 Kwon E-J and Kim M-M: Agmatine modulates melanogenesis via MITF signaling pathway. Eur J Pharmacol 632: 124-130, 2017.

10 Sengupta R, Sheorey SD and Hinge MA: Analgesic and antiinflammatory plants: an updated review. Int J Pharm Sci Rev Res 12: 114-119, 2012.

11 Galgano F, Caruso M, Condelli N and Favati F: Focused review: agmatine in fermented foods. Biogenic Amines in Fermented Foods: 66, 2015.

12 Hansen MB, Nielsen SE and Berg K: Re-examination and further development of a precise and rapid dye method for measuring cell growth/cell kill. J Immunol Methods 119: 203210,1989

13 Kang J-H, Han I-H, Sung M-K, Yoo H, Kim Y-G, Kim J-S, Kawada $\mathrm{T}$ and $\mathrm{Yu}$ R: Soybean saponin inhibits tumor cell metastasis by modulating expressions of MMP-2, MMP-9 and TIMP-2. Cancer Lett 261: 84-92, 2008.

14 Zheng S, Li W, Wang J, Chen Y, Hou W, Gao W, Liu Q and Wang Y: Platycodin D inhibits B16F10 melanoma metastasis via antiangiogenic activity. RSC Adv 6: 10606-10614, 2016.

15 Cheng G, Gao F, Sun X, Bi H and Zhu Y: Paris saponin VII suppresses osteosarcoma cell migration and invasion by inhibiting MMP-2/9 production via the p38 MAPK signaling pathway. Mol Med Rep 14: 3199-3205, 2016.

16 Ma L, Wang R, Nan Y, Li W, Wang Q and Jin F: Phloretin exhibits an anticancer effect and enhances the anticancer ability of cisplatin on non-small cell lung cancer cell lines by regulating expression of apoptotic pathways and matrix metalloproteinases. Int J Oncol 48: 843-853, 2016.
17 Chai J, Luo L, Hou F, Fan X, Yu J, Ma W, Tang W, Yang X, Zhu $\mathrm{J}$ and Kang W: Agmatine reduces lipopolysaccharide-mediated oxidant response via activating PI3K/Akt pathway and upregulating Nrf2 and HO-1 expression in macrophages. PloS one 11: e0163634, 2016.

18 Lee DE, Chung MY, Lim TG, Huh WB, Lee HJ and Lee KW: Quercetin suppresses intracellular ROS formation, MMP activation, and cell motility in human fibrosarcoma cells. J Food Sci 78: H1464-H1469, 2013.

19 Lu X, Duan L, Xie H, Lu X, Lu D, Lu D, Jiang N and Chen Y: Evaluation of MMP-9 and MMP-2 and their suppressor TIMP1 and TIMP-2 in adenocarcinoma of esophagogastric junction. Onco Targets Ther 9: 4343, 2016.

20 Mutlu M, Saatci O, Eyupoglu E, Raza U and Sahin O: Combinatorial targeting of PI3K and MAPK signaling pathways using microRNAs to inhibit tumor growth and metastasis in breast cancer. AACR, 2016. doi: 10.1158/1538-7445.AM20161912

21 Qi C, Xie M, Liang J, Li H, Li Z, Shi S, Yang X, Wang Z, Tang $\mathrm{J}$ and Tang A: Formononetin targets the MAPK and PI3K/Akt pathways to induce apoptosis in human nasopharyngeal carcinoma cells in vitro and in vivo. Int J Clin Exp Med 9: 11801189,2016

22 Su Y, Wan D and Song W: Dryofragin inhibits the migration and invasion of human osteosarcoma U2OS cells by suppressing MMP-2/9 and elevating TIMP-1/2 through PI3K/AKT and p38 MAPK signaling pathways. Anticancer Drugs 27: 660-668, 2016.

23 Li BS, Guo WJ, Hong L, Liu YD, Liu C, Hong SS, Wu DB and Min J: Role of mechanical strain-activated PI3K/Akt signaling pathway in pelvic organ prolapse. Mol Med Rep 14: 243-253, 2016.

24 Hong S, Ngo D-N and Kim M-M: Inhibitory effect of aminoethyl-chitooligosaccharides on invasion of human fibrosarcoma cells. Environ Toxicol Phar 45: 309-314, 2016.

Received August 1, 2017

Revised August 22, 2017

Accepted August 24, 2017 